\title{
A method for obtaining a sparse matrix from the volume integral equation for elastic wave propagation
}

\author{
T. Touhei \\ Department of Civil Engineering, Tokyo University of Science, Japan
}

\begin{abstract}
The advantage of the volume integral equation is that it is possible to clarify the relationship between fluctuations of the wave field and radiation of scattered waves. This paper proposes a method to obtain a sparse matrix for the volume integral equation for elastic wave propagation. The formulation employed here is based on the wavenumber domain solution together with usage of Haar scaling functions. The unitarity of the Fourier transform in terms of the Haar scaling function reveals that the integral equation is transformed into a linear algebraic equation with a sparse matrix. Numerical calculations are carried out to verify the proposed formulation.

Keywords: elastic waves, volume integral equation, sparse matrix, unitary transform, Haar scaling function.
\end{abstract}

\section{Introduction}

Since 1980s, the boundary element technique has been recognized as an efficient tool for the analysis of wave propagation (for example, Brebbia and Walker [3]). On the other hand, the volume integral equation methods have not been used very often except for some cases (for example, Kitahara et al [5]). The advantage of the volume integral equation such as the Lippmann-Schwinger equation (Colton and Kress [4]) is in that it clarifies the relationship between the fluctuation of the medium and the radiation of scattered waves. Standard technique for discretizing the equation, however, leads to a large and dense matrix for the volume integral equation, that makes sometimes numerical analysis impossible even by recent high performance computers. 
In this paper, a method for obtaining a sparse matrix from the volume integral equation is presented. The method is based on the usage of the Fourier transform and the Haar scaling function (Williams and Amaratunga [7]). The volume integral equation in the wavenumber domain is discretized by means of the Haar scaling function. The unitarity of the Fourier transform shows that the Fourier inverse transform of the Haar scaling functions form the orthonormal basis and as a result, a sparse matrix is found to be derived from the volume integral equation in the case that the spectral structure of the fluctuation of the wave field is narrow band. Several numerical calculations are carried out to verify the accuracy of the present method.

\section{Theoretical formulation}

\subsection{Basic equations}

An elastic full space of three dimension is considered in this paper. The elastic wave field is assumed to have a fluctuation represented by the Lamé constants such that

$$
\begin{aligned}
& \lambda(x)=\lambda_{0}+\tilde{\lambda}_{1}(x) \\
& \mu(x)=\mu_{0}+\tilde{\mu}_{1}(x), \quad\left(x \in \mathbb{R}^{3}\right)
\end{aligned}
$$

where $\lambda_{0}$ and $\mu_{0}$ are the background Lamé constants and $\tilde{\lambda}$ and $\tilde{\mu}$ are their fluctuations. Note that $x \in \mathbb{R}^{3}$ denotes the spatial point. The governing equation for the elastic wave propagation for the medium can be derived according to the literature (for example, Aki and Richards [1]). The governing equation is expressed by

$$
\left(\lambda_{0}+\mu_{0}\right) \partial_{i} \partial_{j} u_{j}+\mu_{0} \partial_{k} \partial_{k} u_{i}+\rho \omega^{2} u_{i}=N_{i j} u_{j}
$$

where $u_{i}$ is the displacement field whose subscript denotes the component of the Cartesian coordinate, $\rho$ is the mass density, $\omega$ is the circular frequency, $\partial$ is the partial differential operator whose subscript denotes the parameter for the differentiation and $N_{i j}$ is the operator describing the fluctuation of the elastic medium. The summation convention is applied to the subscript index for the component of the coordinate. The explicit form of $N_{i j}$ is expressed by

$$
\begin{aligned}
N_{i j}= & -(\tilde{\lambda}(x)+\tilde{\mu}(x)) \partial_{i} \partial_{j}-\delta_{i j} \tilde{\mu}(x) \partial_{k} \partial_{k} \\
& -\partial_{i} \tilde{\lambda}(x) \partial_{j}-\delta_{i j} \partial_{k} \tilde{\mu}(x) \partial_{k}-\partial_{j} \tilde{\mu}(x) \partial_{i}
\end{aligned}
$$

where $\delta_{i j}$ is the Kronecker delta. The volume integral equation for the elastic wave field is directly derived from Eq. (2), which is

$$
u_{i}(x)=f_{i}(x)-\int_{\mathbb{R}^{3}} g_{i j}(x, y) N_{j k}(y) u_{k}(y) d y
$$


where $f_{i}$ is the plane incident wave and $g_{i j}(x, y)$ is the Green's function expressed by

$$
\begin{aligned}
g_{i j}(x, y)= & \frac{1}{4 \pi \mu_{0}} \delta_{i j}\left(Z_{1}^{(T)}(r)+Z_{2}^{(T)}(r)-\left(c_{T} / c_{L}\right)^{2} Z_{2}^{(L)}(r)\right) \\
& +\frac{1}{4 \pi \mu_{0}} \partial_{i} r \partial_{j} r\left(Z_{3}^{(T)}(r)-\left(c_{T} / c_{L}\right)^{2} Z_{3}(r)\right)
\end{aligned}
$$

In Eq. (5), $r=|x-y|$ and $Z_{j}^{(p)}(r), j=1,2,3, p=T, L$ is the function defined by

$$
\begin{aligned}
& Z_{1}^{(p)}(r)=\frac{1}{r} \exp \left(i k_{p} r\right) \\
& Z_{2}^{(p)}(r)=-\frac{1}{r}\left(\frac{1}{k_{p}^{2} r^{2}}+\frac{i}{k_{p} r}\right) \exp \left(i k_{p} r\right) \\
& Z_{3}^{(p)}(r)=-Z_{1}^{(p)}(r)-3 Z_{2}^{(p)}(r)
\end{aligned}
$$

where $k_{T}$ and $k_{L}$ are the wavenumber of the $\mathrm{S}$ and $\mathrm{P}$ waves, respectively.

\subsection{Fourier transform of Integral equation}

The formulation presented here employs the Fourier integral transform for the volume integral equation. The Fourier and its inverse integral transforms (Reed and Simon [6]) are respectively expressed as

$$
\begin{aligned}
& \hat{f}(\xi)=\frac{1}{\sqrt{2 \pi}^{3}} \int_{\mathbb{R}^{3}} f(x) \exp (-i \xi \cdot x) d x \\
& \check{f}(x)=\frac{1}{\sqrt{2 \pi}^{3}} \int_{\mathbb{R}^{3}} f(\xi) \exp (i \xi \cdot x) d \xi
\end{aligned}
$$

where $\hat{f}$ denotes the Fourier transform of $f$ and $\check{f}$ denotes the Fourier inverse transform of $f$. Note that $\xi \in \mathbb{R}^{3}$ is used for the point in the wavenumber space. The Fourier transform of the volume integral equation in terms of scattered wave $v_{i}(x)=u_{i}(x)-f_{i}(x)$ becomes as

$$
\begin{gathered}
\hat{v}_{i}(\xi)=-\hat{h}_{i j}(\xi) \hat{q}_{j}\left(\xi-\xi_{p}\right)-\hat{h}_{i j}(\xi) \hat{w}_{j}(\xi) \\
w_{j}(x)=N_{j k}(x) v_{k}(x)
\end{gathered}
$$

where $\hat{h}_{i j}(\xi)$ is related to the Fourier transform of the Green's function that is expressed by

$$
\begin{aligned}
\hat{h}_{j}(\xi)= & \frac{\delta_{i j}}{\mu_{0}\left(|\xi|^{2}-k_{T}^{2}+i \epsilon\right)} \\
& -\frac{\xi_{i} \xi_{j}}{2 \mu_{0}(1-\nu)\left(|\xi|^{2}-k_{T}^{2}+i \epsilon\right)\left(|\xi|^{2}-k_{L}^{2}+i \epsilon\right)}
\end{aligned}
$$


Note that $\epsilon$ in Eq. (10) is the infinitesimal positive number and $\nu$ is the Poisson ratio. In addition, $\xi_{p}$ in Eq.(8) is the wavenumber vector of the plane incident wave and $\hat{q}$ is the function related to the fluctuation of the medium and plane incident wave.

\subsection{Discretization of the volume integral equation}

The Fourier transformed volume integral equation shown in Eq. (8) can be discretized by means of the Haar scaling functions. Let $\left\{\phi_{\alpha}(\xi)\right\}_{\alpha=1}^{N}$ is the set of the Haar scaling functions embedded in the wavenumber space, where $\alpha$ is the integer to identify the element of the set of the Haar scaling functions. In the following formulation, the Greek character used for the subscript is for identifying the Haar scaling functions. For each $\alpha, \phi_{\alpha}$ has the resolution and integer shift vector such that

$$
\phi_{\alpha}(\xi)=2^{m_{\alpha} / 2} \prod_{j=1}^{3} \phi\left(2^{m_{\alpha}} \xi_{j}-\Gamma_{j(\alpha)}\right)
$$

where $m_{\alpha}$ is the resolution, $\Gamma_{j(\alpha)}, j=1,2,3$ is the component of the integer shift vector and $\phi$ is the Haar box function such that

$$
\phi(x)=\left\{\begin{array}{c}
1(0 \leq x<1) \\
0 \text { otherwise }
\end{array} \quad(x \in \mathbb{R})\right.
$$

The set of the Haar scaling functions is set up so that the support of each element is disjoint each other. Namely,

$$
\operatorname{supp} \phi_{\alpha}(\xi) \cap \operatorname{supp} \phi_{\beta}(\xi)=\emptyset(\alpha \neq \beta)
$$

In addition, the set of the Haar scaling function fills a region in the wavenumber space $C$ densely,

$$
C \backslash \bigcup_{\alpha=1}^{N} \operatorname{supp} \phi_{\alpha}=\emptyset
$$

The region $C$ is set up such that

$$
C=\left\{\xi=\left(\xi_{1}, \xi_{2}, \xi_{3}\right) \mid-L \leq \xi_{j} \leq L, j=1,2,3\right\}
$$

where $L$ is the positive number, which is taken large enough for the numerical calculation. Due to the above properties, the set of the Haar scaling functions forms the orthogonal basis in the wavenumber domain. Namely,

$$
\left\langle\phi_{\alpha}(\xi), \phi_{\beta}(\xi)\right\rangle=\delta_{\alpha \beta}
$$

where $\langle\cdot, \cdot\rangle$ is the scalar product of the functions defined by

$$
\langle f(\xi), g(\xi)\rangle=\int_{\mathbb{R}^{3}} f^{*}(\xi) g(\xi) d \xi
$$


Note that the Fourier inverse transform of the Haar scaling functions also form the orthonormal basis according to the unitarity of the Fourier transform. Therefore, the following equation can be established:

$$
\left\langle\check{\phi}_{\alpha}(x), \check{\phi}_{\beta}(x)\right\rangle=\delta_{\alpha \beta}
$$

where $\check{\phi}_{\alpha}$ is the Fourier inverse transform of the Haar scaling function:

$$
\begin{aligned}
\check{\phi}_{\alpha}(x)= & \frac{1}{{\sqrt{2^{m_{\alpha}+1} \pi}}^{3}} \times \\
& \prod_{k=1}^{3} \frac{\sin \theta_{k(\alpha)}}{\theta_{k(\alpha)}} \exp \left(i \theta_{k(\alpha)}\left(2 \Gamma_{k(\alpha)}+1\right)\right)
\end{aligned}
$$

where

$$
\theta_{k(\alpha)}=\frac{x_{k}}{2^{m_{\alpha}+1}}
$$

At this stage, we are in a situation in that we can discretize the integral equation (8). We expand each variable of Eq. (8) such that

$$
\begin{aligned}
\hat{v}_{i}(\xi) & =\sum_{\alpha} \hat{V}_{i(\alpha)} \phi_{\alpha}(\xi) \\
\hat{h}_{i j}(\xi) & =\sum_{\alpha} \hat{H}_{i j(\alpha)} \phi_{\alpha}(\xi) \\
\hat{q}_{j}(\xi) & =\sum_{\alpha} \hat{Q}_{j(\alpha)} \phi_{\alpha}(\xi) \\
\hat{w}_{j}(\xi) & =\sum_{\alpha} \hat{W}_{j(\alpha)} \phi_{\alpha}(\xi)
\end{aligned}
$$

Then, Eq. (8) can be modified into

$$
\hat{V}_{i(\alpha)}=-c_{\alpha} \hat{H}_{i j(\alpha)} \hat{Q}_{j(\alpha)}-c_{\alpha} \hat{H}_{i j(\alpha)} \hat{W}_{j(\alpha)}
$$

For Eq. (22), the following equation:

$$
\phi_{\alpha}(\xi) \phi_{\beta}(\xi)=c_{\alpha} \delta_{\alpha \beta}
$$

where

$$
c_{\alpha}=\left(2^{m_{\alpha} / 2}\right)^{3}
$$

is used. To discretize Eq. (9), the Fourier inverse transform of $\hat{v}_{i}$ and $\hat{w}_{j}$ which has the following forms

$$
\begin{aligned}
v_{i}(x) & =\sum_{\alpha} \hat{V}_{i(\alpha)} \check{\phi}_{\alpha}(x) \\
w_{j}(x) & =\sum_{\alpha} \hat{W}_{j(\alpha)} \check{\phi}_{\alpha}(x)
\end{aligned}
$$


are used. Substituting Eq. (25) into Eq. (9) as well as using the orthogonality relation shown in Eq. (18) leads to the following equation:

$$
\hat{W}_{i(\alpha)}=S_{i j(\alpha \beta)} \hat{V}_{j(\beta)}
$$

where $S_{i j(\alpha \beta)}$ is due to the following operation:

$$
S_{i j(\alpha \beta)}=\left\langle\check{\phi}_{\alpha}, N_{i j} \check{\phi}_{\beta}\right\rangle
$$

As a result, the following linear algebraic equation in terms of $\hat{V}_{i(\alpha)}$ is derived:

$$
\hat{V}_{i(\alpha)}=F_{i(\alpha)}-A_{i k(\alpha \beta)} \hat{V}_{k(\beta)}
$$

where

$$
\begin{aligned}
F_{i(\alpha)} & =-c_{\alpha} \hat{H}_{i j(\alpha)} \hat{Q}_{j(\alpha)} \\
A_{i k(\alpha \beta)} & =c_{\alpha} \hat{H}_{i j(\alpha)} S_{j k(\alpha \beta)}
\end{aligned}
$$

The scattered wave field can be derived by solving Eq. (28), since we have

$$
v_{i}(x)=\sum_{\alpha} \hat{V}_{i(\alpha)} \check{\phi}(x)
$$

At the end of the formulation, note that the operation of the product shown in Eq. (27) can be carried out without difficulty. To carry out the operation, the fluctuation of the lamé constants $\tilde{\lambda}$ and $\tilde{\mu}$ are expanded such that

$$
\begin{aligned}
\tilde{\lambda}(x) & =\sum_{\beta \in B} \Lambda_{\beta} \check{\phi} \\
\partial_{j} \tilde{\lambda} & =\sum_{\beta \in B} \Lambda_{\beta}^{(j)} \check{\phi}(x) \\
\tilde{\mu}(x) & =\sum_{\beta \in B} M_{\beta} \check{\phi} \\
\partial_{j} \tilde{\mu} & =\sum_{\beta \in B} M_{\beta}^{(j)} \check{\phi}(x)
\end{aligned}
$$

where $B$ is the set of index for expressing the fluctuation of the Lamé constants.

Substituting Eq, (31) into Eq. (27) clarifies that the operation for Eq. (27) can be constituted by the following integral formulas:

$$
S_{i j(\alpha \gamma)}=\sum_{\beta \in B} \sum_{p} \gamma_{p} \int_{\mathbb{R}^{3}} \check{\phi}_{\alpha}^{*}(x) \check{\phi}_{\beta}(x) \partial^{p} \check{\phi}_{\gamma}(x) d x
$$

where $p=\left(p_{1}, p_{2}, p_{3}\right)$ and $\partial^{p}=\partial_{1}^{p_{1}} \partial_{2}^{p_{2}} \partial_{3}^{p_{3}}$. In addition, $\gamma_{p}$ in Eq. (32) is uniquely determined by $N_{i j}$ and coefficients $\Lambda_{\beta}, \Lambda_{\beta}^{(j)}, M_{\beta}$ and $M_{\beta}^{(j)}$ in Eq. (31). The closed form of the result of the integral of Eq. (32) is possible, which leads to the fact that the matrix $S_{i j(\alpha \gamma)}$ is sparse in the case that the range of set of $B$ is narrow. Namely, in the case that the spectral structure of the fluctuation of the Lamé constants is narrow band, a sparse matrix is obtained. 


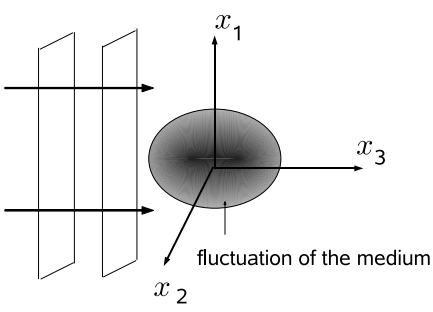

Plane Incident wave

Figure 1: Analyzed model.

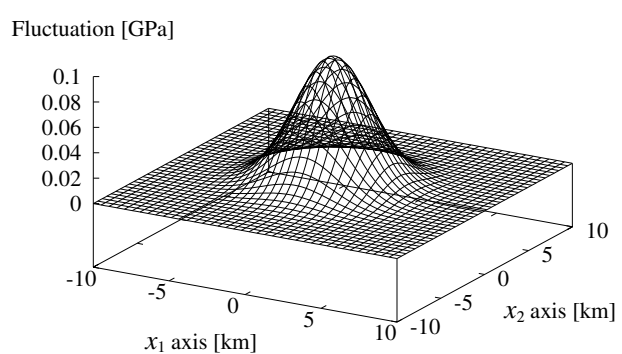

Figure 2: Fluctuation of the Lamé constants.

\section{Numerical examples}

\subsection{Analyzed model}

The concept of the analyzed model is shown in Figure 1, in that the plane incident wave is propagating to the fluctuated area along $x_{3}$ axis. The background Lamé constants are set at $\lambda_{0}=2[\mathrm{GPa}], \mu_{0}=1[\mathrm{GPa}]$ and the mass density of the wave field is $\rho=1\left[\mathrm{~g} / \mathrm{cm}^{3}\right]$. In addition, the frequency of the wave field is $1[\mathrm{~Hz}]$. Therefore, the $\mathrm{P}$ wavenumber of the background wave field is $k_{L}=3.14\left[\mathrm{~km}^{-1}\right]$ and the $\mathrm{S}$ wavenumber of that is $k_{T}=6.28\left[\mathrm{~km}^{-1}\right]$. The fluctuation of the Lamé constants are set at

$$
\begin{aligned}
& \tilde{\lambda}(x)=0.1 \exp \left(-0.1|x|^{2}\right) \\
& \tilde{\mu}(x)=0.1 \exp \left(-0.1|x|^{2}\right) \quad[\mathrm{Gpa}]
\end{aligned}
$$

The fluctuation of Lamé constant at $x_{3}=0$ plane is shown in Figure 2. As shown in Figure 2, the fluctuation gradually decreases towards the far field range. The Fourier transform of the fluctuation of the Lamé constants for Eq. (33) becomes

$$
\hat{\tilde{\lambda}}(\xi)=\hat{\tilde{\mu}}(\xi)=\frac{1}{\sqrt{2 \eta}^{3}} \exp \left(-|\xi|^{2} /(4 \eta), \quad(\eta=0.1)\right.
$$

As can be seen in the following, the spectral structure of the fluctuation of the Lamé constants is narrow enough to generate a sparse matrix.

\subsection{Haar scaling functions used for the analysis}

As shown in the formulation, the volume integral equation is discretized by the Haar scaling functions in the wavenumber space. Figures 3 to 6 are the location of the Haar scaling functions in the wavenumber space, where $s_{j}, j=1,2,3$ indicates the dimensionless wavenumber defined by $s_{j}=\xi / k_{T}$. The wavenumber space is spanned by the Haar basis for the region $-10 \leq s_{j} \leq 10(j=1,2,3)$. 


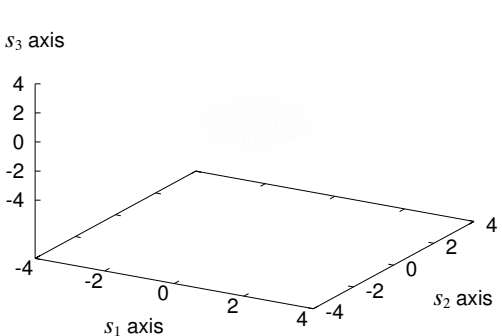

Figure 3: Haar scaling functions in the wavenumber space $(m=1 \sim$ $3)$.

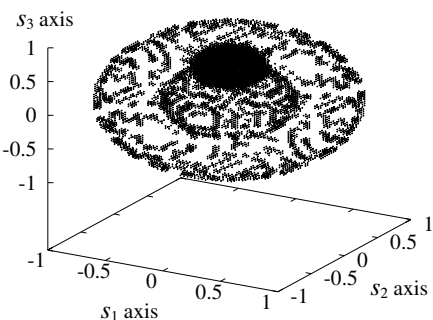

Figure 5: Haar scaling functions in the Figure 6: Haar scaling functions in the wavenumber space $(m=5)$.

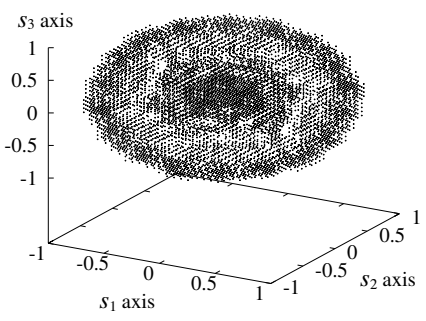

Figure 4: Haar scaling functions in the wavenumber space $(m=4)$.

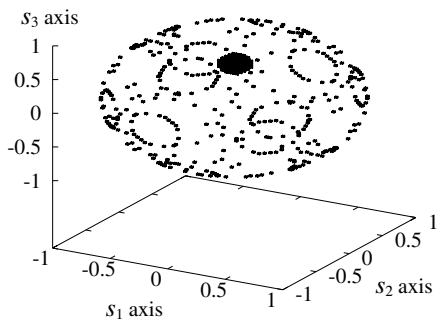

wavenumber space $(m=6)$.

The resolution $m$ ranges from 1 to 6 , that is for the dimensionless wavenumber. In these figures, the center of the supports of the Haar scaling functions are plotted. The size of the supports become smaller as the resolution increases. As a result, the resolution of the Haar basis is described by the density of the points. The higher resolution of the Haar basis is used for the region close to singular point of the Green's function in the wavenumber domain. To simplify the view of the location of the Haar basis, the Haar basis is shown in the region $-4 \leq s_{j} \leq 4(j=1,2,3)$ in figure 3 . Note that the resolution of the Haar scaling functions that located farthest outside the region is $m=1$. The number of the Haar scaling functions used for the analysis here are 97824.

\subsection{Sparse matrix obtained from the present procedure}

Figure 7 shows the structure of the matrix obtained form the Haar scaling functions shown in Figures 3-6. In figure 7, the non-zero elements are plotted. Those non-zero elements are judged from Eq. (32). The size of the matrix is about $290,000 \times 290,000$ with the sparse ratio $2.8 \%$. To solve the linear algebraic 


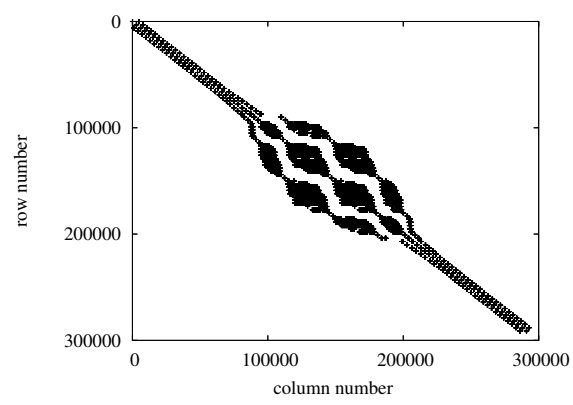

Figure 7: Structure of the matrix.

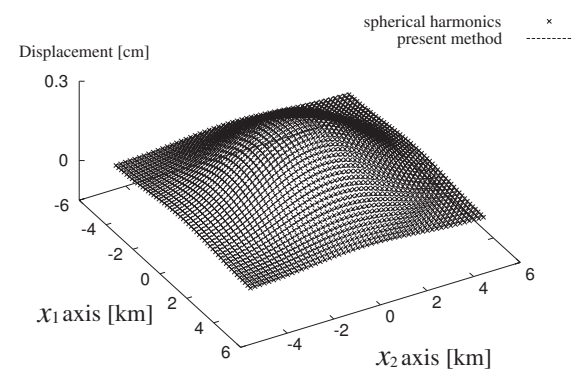

Figure 9: Comparison of displacement Figure 10: Displacements in $x_{1}-x_{3}$ at $x_{3}=2 \mathrm{~km}$.

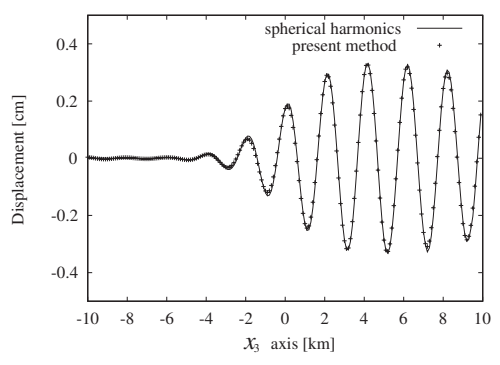

Figure 8: Comparison of displacement.

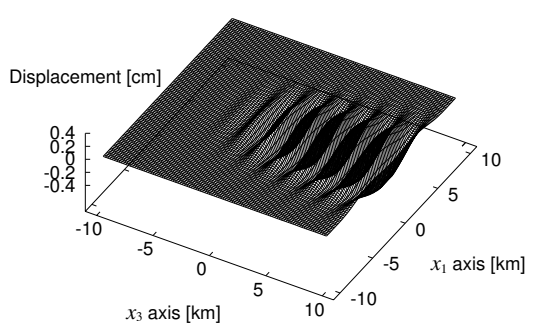

plane.

equation, the iterative scheme of Bi-CGSTAB method (Barrett et al [2]) is employed. The elapsed time for solving the matrix was about 5 minutes by IBM pSeries 690 provided by Tokyo University of Science, for the case of 32-cpu parallel processing. The number of iteration for the convergence of the solution was three. The condition for the convergence of the linear algebraic equation $\boldsymbol{A} \boldsymbol{x}=\boldsymbol{b}$ is $|\boldsymbol{A} \boldsymbol{y}-\boldsymbol{b}|<0.001|\boldsymbol{b}|$, where $\boldsymbol{A}$ is the coefficient matrix and $\boldsymbol{y}$ is the approximate solution.

\subsection{Properties of the scattered wave field}

Now, assume that the plane incident wave propagating along $x_{3}$ axis is $\mathrm{P}$ wave. The comparison of the displacement of the scattered wave field between the present method and the spherical harmonics expansion is shown in figure 8 . In the figure, the displacement component of $x_{3}$ direction are compared along $x_{3}$ axis. Good agreements can be found in both two methods in figure 8, which validates the accuracy of the present method. The amplitude of the backward scattering is found to be very small in figure 8 , while that of the forward scattering is relatively large and decreases slowly towards the far field region. The slow decrease of the displacement amplitude is due to the slow decrees of the fluctuation of the 
Lamé constants towards the far field region, which is shown in figure 2. Figure 9 shows the distribution of the displacement amplitude at $x_{3}=2 \mathrm{~km}$, in which the displacement amplitude is also compared with the spherical harmonics expansion. As can be seen in figure 9, the peak value of the displacement amplitude is found in the origin of the $x_{3}$ plane. The displacement slowly decreases toward the far field region. The direction of the displacement is outstanding in the $x_{3}$ component, which indicates that the scattered waves are mainly constituted by $\mathrm{P}$ wave. Figure 10 shows the distribution of the displacement of $x_{3}$ component in $x_{1}-x_{3}$ plane. It is found from figure 10 that the scattered wave does not spread widely in the forward region, indicating that the scattered waves here has rather strong directionality.

\section{Conclusion}

A method for obtaining a sparse matrix was presented in this paper for the volume integral equation. The Fourier transform was employed to the volume integral equation. The usage of the Haar scaling functions in the wavenumber domain as well as the unitarity of the Fourier transform revealed that a sparse matrix was derived from the volume integral equation. A iterative scheme for solving the linear algebraic equation was found to be successfully applied to the sparse matrix. Numerical results ensured the validity and accuracy of the present method.

\section{References}

[1] Aki, K. and Richards, P.G. (1980): Quantitaive Seismology. Theory and Methods, W.H. Freeman and Comnpany.

[2] Barrett, M., Berry, M., Chan,T.F., Demmel, J., Donato, J. M., Dongarra, J., Eijkhout, V., Pozo,R., Romine, C. and Van der Vorst, H. (1994). Templates for the solution of Linear Systems: Building Blocks for Iterative Methods, SIAM.

[3] Brebbia, C. A. and Walker, S. (1980). Boundary element techniques in engineering. London, Butterworth and Co. Ltd.

[4] Colton, D. and Kress, R. (1998). Inverse acoustic and electromagnetic scattering theory, Berlin, Springer.

[5] Kitahara, M., Niwa, Y., Hirose, S. and Yamazaki, M. (1984). Coupling of numerical Green's matrix and boundary integral equations for the elastodynamic analysis of inhomogeneous bodies on an elastic half-space, Applied Mathematical Modelling, 8, 397-407.

[6] Reed, M. and Simon, B. (1975). Method of Modern Mathematical Physics, Vol. II, Fourier Analysis and Self-adjointness, Academic Press.

[7] Williams, J.R. and Amaratunga, K.: Introduction to wavelets in engineering, International Journal for Numerical Methods in Engineering, Vol. 37, pp. 2365-2388, 1994. 